\title{
CARDIOVASCULAR MANIFESTATIONS OF HYPOTHYROIDISM
}

\author{
E. V. S. Maben ${ }^{1}$, Shilpa Mulki
}

1 Professor and HOD, Department of General Medicine, A. J. Institute of Medical Sciences, Mangalore, Karnataka, India. ${ }^{2}$ Assistant Professor, Department of General Medicine, A. J. Institute of Medical Sciences, Mangalore, Karnataka, India.

\begin{tabular}{l}
\hline ABSTRACT \\
BACKGROUND \\
Subclinical thyroid disease, hyper and hypothyroidism are very common entities that manifest in thyroid dysfunction. The \\
importance of mild thyroid dysfunction has not been adequately assessed so far. This had led to lack of uniformity in approach and \\
treatment of subclinical hypothyroidism. Nearly 108 million people in India suffer from endocrine, as well as metabolic disorders. \\
The most frequently seen are diabetes and thyroid dysfunction. Thyroid dysfunction is most frequently seen in women.1,2 Studies of \\
the goitrous subjects showed overall prevalence of hypothyroidism to be $5.4 \%$. The need for treatment of subclinical \\
hypothyroidism, is a highly questioned one. This study aims at proving reversibility of cardiovascular involvement, in subclinical \\
hypothyroidism, with apt treatment.
\end{tabular}

\section{METHODS}

This study was conducted as a prospective cohort study, by convenient sampling. 32 newly diagnosed consenting patients with subclinical and overt hypothyroidism, in the age group of 15-50 years. Those excluded were known to have cardiac disease, and/or diabetes. On initial assessment, symptoms with examination findings were noted as per the proforma. They were investigated with regard to parameters such as Fasting Lipid profile, 12 lead ECG and 2D Echo at presentation, during the $3^{\text {rd }}$ and $6^{\text {th }}$ month follow up, during which period they were on treatment with Levothyroxine substitution therapy based on their body weight.

\section{RESULTS}

Echo cardiographic findings, i.e., diastolic dysfunction, seen in $15.6 \%$ of the patients, improved with L thyroxine therapy in only $12.5 \%$ of the population. Other findings e.g. raised interventricular septal dimensions and pericardial effusions did not show any significant changes in spite of treatment.

\section{CONCLUSIONS}

On the part of the treating physician, clinical inertia with regard to treatment of subclinical hypothyroidism, should not be encouraged. 32 newly diagnosed hypothyroid patients presented with an abundance of features suggestive of raised cardiovascular morbidity in the form of raised fasting lipids, bradycardia, raised interventricular septal dimensions and diastolic dysfunction. Treatment in the form of levothyroxine therapy improved all the above-mentioned parameters to a small extent, and other studies have in fact, shown a long-term benefit. This study proves the need to diagnose and treat hypothyroidism at an early stage. It requires to be done on a large-scale basis with follow up for a prolonged duration to reiterate the benefits of treatment of hypothyroidism.

\section{KEY WORDS}

Subclinical Hypothyroidism, Reversible Cardiovascular Disease, Diastolic Dysfunction, L Thyroxine. ECG, 2D Echocardiography HOW TO CITE THIS ARTICLE: Maben EVS, Mulki S. Cardiovascular manifestations of hypothyroidism. J. Evolution Med. Dent. Sci. 2019;8(28):2260-2263, DOI: $10.14260 /$ jemds/2019/495

\section{BACKGROUND}

Nearly 108 million people in India suffer from endocrine, as well as metabolic disorders. The most frequently seen are diabetes, and thyroid dysfunction. Thyroid dysfunction is most frequently seen in women.1,2 Studies of the goitrous subjects showed overall prevalence of hypothyroidism in $5.4 \%{ }^{3}$ Amongst the manifestations of hypothyroidism, overt and subclinical forms may manifest. Overt hypothyroidism refers to cases in which the serum thyroid stimulating hormone (TSH) concentration is elevated and serum free thyroxine is below the reference range.

'Financial or Other Competing Interest': None.

Submission 13-05-2019, Peer Review 25-06-2019,

Acceptance 03-07-2019, Published 15-07-2019.

Corresponding Author:

Dr. E. V.S. Maben

Professor and HOD,

Department of General Medicine,

A. J. Institute of Medical Sciences,

Mangalore, Karnataka, India.

E-mail: suranjanmaben@hotmail.com

DOI: $10.14260 /$ jemds/2019/495
Subclinical hypothyroidism is defined as a serum thyroid stimulating hormone (TSH) above the defined upper limit of the reference range, with a serum free thyroxine (T4) within the reference range. The progression to overt hypothyroidism is approximately 2-5\% per year. ${ }^{4}$ Thyroid hormones exert direct effects on almost all tissues of the body. It causes multi organ dysfunction due to deranged metabolism. The cardiac dysfunction ranges from functional systolic/diastolic dysfunction to overt failure and coronary artery disease. This is as a result of the direct effect of the disease. Hypothyroidism is also typically characterized by hypercholesterolemia and a marked increase in low-density lipoprotein and apolipoprotein B. This occurs secondary to a decreased fractional clearance of low-density lipoproteins by a reduced number of low-density lipoproteins receptors in the liver. 5

This study highlights the cardiovascular complications, as they are some of the most profound and reproducible clinical findings associated with thyroid disease. It assesses the CVS parameters in newly diagnosed hypothyroid patients, and it's reversal with treatment. There are only few studies done in our country to assess the same. This study will also re-iterate the need to have a high degree of clinical suspicion for 
hypothyroidism, as early treatment, in the form of L-thyroxine therapy, can bring about reversibility of the cardiovascular changes brought about by the disease.

\section{Aim of The Study}

The need for treatment of subclinical hypothyroidism, is a highly questioned one. This Study aims at proving reversibility of cardiovascular involvement, in subclinical hypothyroidism, with apt treatment.

\section{METHODS}

After Clearance from the Institutional Ethical Committee, this prospective study was conducted as a prospective study, by convenient sampling. 32 newly diagnosed patients with subclinical and overt hypothyroidism, between the age of 1550 years, who provided written consented were included. Those excluded were known to have cardiac disease, and/or diabetes. On initial assessment, symptoms with examination findings were noted as per the proforma. They were investigated on parameters such as Fasting Lipid profile (Bioless Automated Machine), 12 lead ECG (BPL Machine) and 2D Echo (Utilising the Phillips Hd11xe). These parameters were evaluated at first contact, then during the $3^{\text {rd }}$ and $6^{\text {th }}$ month follow up, during which period they were on treatment with Levothyroxine substitution therapy based on their body weight.

\section{Statistical Analysis}

The data was collected and analysed using standard statistical chi - square test, $\mathrm{P}<0.05$ statistically significant. Data was entered in Microsoft excel and analysis was done using SPSS version 22

\section{RESULTS}

In males, the most common age group in which hypothyroidism was encountered was 21-25 years, whereas amongst the females, ages 31-35 years were more frequently found to have hypothyroidism. Among the sexes, in concordance to many studies, females had a higher incidence of hypothyroidism than men.

\section{Symptoms at Presentation}

Frequent symptoms seen in the study population included hoarse voice amongst the male population in the study, although with no statistical significance and weight gain in the female population, which was the most statistically significant symptom. Menorrhagia was found to be present in $38.9 \%$ of the female patients. Amongst males, a BMI of 20-24.9 was commonest, whereas in females a BMI of 25 -29.9 was most commonly found. "Prolonged relaxation of deep tendon reflexes" was the commonest finding amongst males during examination, well in concordance with previous studies by Lambert and Underdahl. ${ }^{6} \mathrm{Cool}$ peripheral extremities were the commonest finding in the female strata of this study. Statistically, "prolonged relaxation of deep tendon reflexes" is more frequent in male, than female patients. Most participants in the study presented with goitre. With respect to presence vs. absence of goitre amongst the total population, the difference was not statistically significant. ( $p$ value $=0.677$ ).

\begin{tabular}{|c|c|c|c|c|c|}
\hline Symptoms & Male & $\mathbf{\%}$ & Female & $\mathbf{\%}$ & p Value \\
\hline Weight Gain & 7 & 50 & 15 & 83.3 & 0.037 \\
\hline Cold Intolerance & 4 & 28.6 & 9 & 50 & 0.289 \\
\hline Hoarse Voice & 11 & 78.6 & 9 & 50 & 0.147 \\
\hline Poor Memory & 8 & 57.1 & 11 & 61.1 & 0.821 \\
\hline Muscle Cramping & 6 & 42.9 & 4 & 22.2 & 0.267 \\
\hline Menorrhagia & 0 & 0 & 7 & 38.9 & 0.01 \\
\hline \multicolumn{7}{|c|}{ Table 1. Symptoms at Presentation } \\
\hline
\end{tabular}

\begin{tabular}{|c|c|c|c|}
\hline Present & $\begin{array}{c}\text { Wayne } \\
(\mathbf{1 9 6 0 )})^{22}\end{array}$ & $\begin{array}{c}\text { Anil Kumar et al } \\
(\mathbf{1 9 8 7})^{\mathbf{7 2}}\end{array}$ & $\begin{array}{c}\text { William FC et al } \\
(\mathbf{1 9 7 7})^{\mathbf{7 1}}\end{array}$ \\
\hline $47 \%$ & $8 \%$ & $14.3 \%$ & $13.3 \%$ \\
\hline \multicolumn{4}{|c|}{ Table 2. Comparison of Bradycardia in the Preset with Past Studies } \\
\hline
\end{tabular}
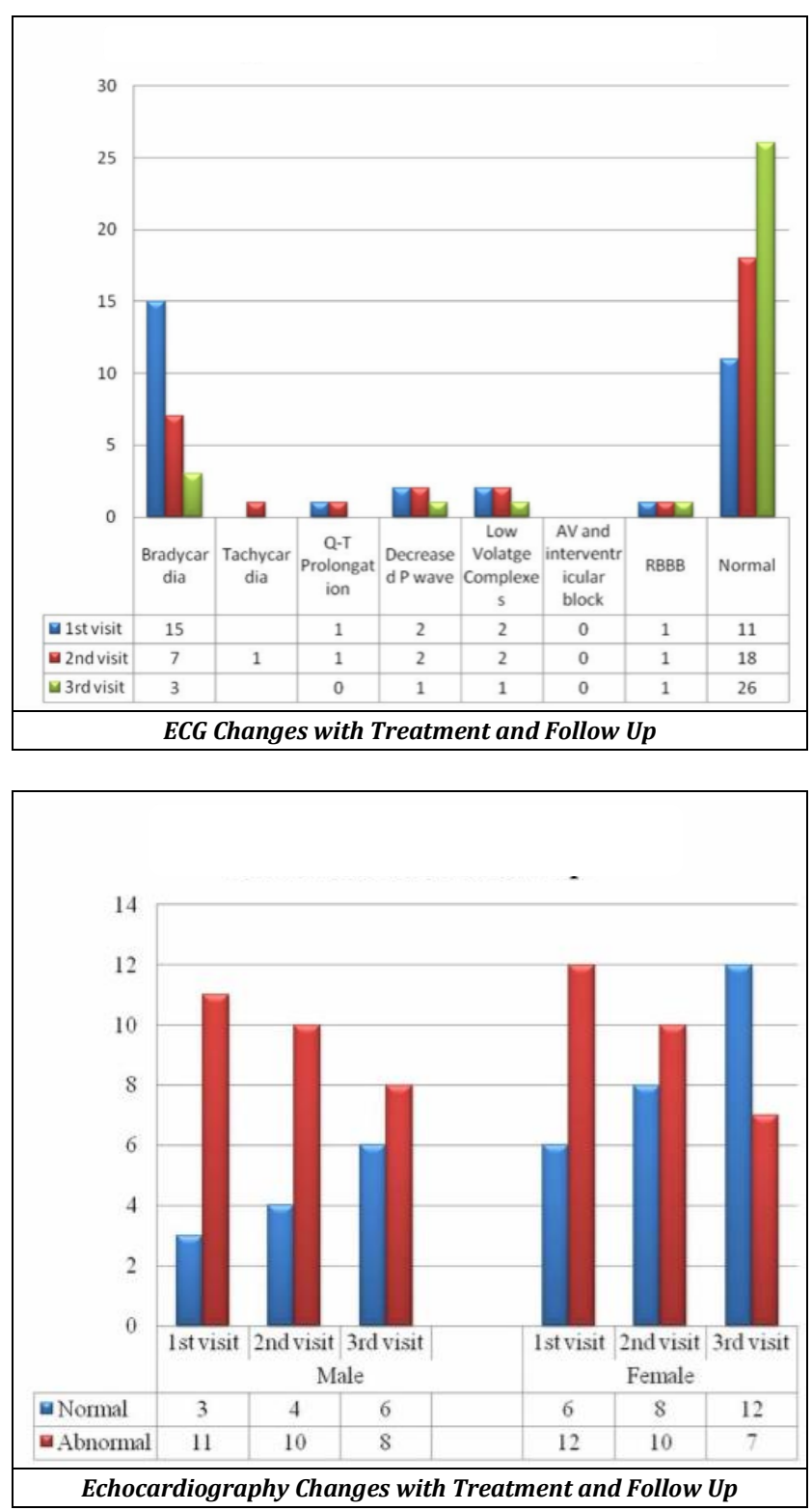

Serum cholesterol levels of 150-199 and $>200 \mathrm{mg} / \mathrm{dl}$ was commonest amongst the male. In females, a similar range of 150-199 mg/dl was encountered during their first visit. Treatment in males after treatment with L-thyroxine therapy, improved the serum cholesterol levels to between 100-149 $\mathrm{mg} / \mathrm{d}$. In females, treatment of hypothyroid state reduced the serum cholesterol in most to levels $<100 \mathrm{mg} / \mathrm{d}$. In the male subset, the improvement was found to be statistically significant $(\mathrm{p}$ value $=0.01)$, unlike in the female subset $(\mathrm{p}$ value $=0.239$ ). Both male and female subsets of the study 
observed an improvement in serum triglycerides with L thyroxine substitution therapy. ( $\mathrm{p}$ value $=0$ for both groups).

In the present study amongst males, and females alike, bradycardia was the commonest finding during the $1^{\text {st }}$ visit. Decreased $\mathrm{P}$ wave is seen in 2 patients in this study. Low voltage complexes were seen one male, and one female patient in the current study, both were proven to have pericardial effusion on echocardiography. RBBB was seen in one male patient, an ECG finding that persisted throughout follow up period. Bradycardia was the only parameter seen to revert back to a normal ECG in more male and female patients with statistical significance. Initial assessment revealed a normal Echocardiography in $16.7 \%$ males and $33.3 \%$ females. With treatment, totally $43 \%$ males had a normal echo at the end of 6 months treatment, and total of $66.7 \%$ female patients had a normal echo study by the end of 6 months of treatment with Levothyroxine therapy. The number of normal Echo reports increased with time and treatment as evidenced by a palue of 0.34 amongst the female patients. Other parameters did not show a significant change with treatment.

\section{DISCUSSION}

The study population consisted of males and females between the ages of 15-50 years to ensure age related cardiac changes do not interfere with the objective (Reversible cardiac dysfunction while on treatment with L-thyroxine therapy). Overall, there was preponderance of female patients consisting of $56.3 \%$ of total study population. This is well in concordance to Harrison's Principles of Internal Medicine; which quotes that "The mean annual incidence rate of hypothyroidism is up to 4 per 1000 women and 1 per 1000 men."

The most common symptoms included hoarse voice and poor memory amongst the male population in the study, although with no statistical significance. In the female population, weight gain was the most statistically significant symptom. Menorrhagia was found to be present in 38.9\% of the female patients. Many studies disclose that amongst the common symptoms, hoarseness of voice, slowness and poor memory are amid the primary symptoms that patients present to the physician.7,8 Menorrhagia was found to account for 20.1 $\%$ of hypothyroid patients who were being worked up for infertility; a common menstrual abnormality amongst hypothyroid females in a study by Fauzia Imtiaz et al. ${ }^{9}$

Data collected in "The Multiple Risk Factor Intervention Trial" ( MRFIT ), was of high precision and showed that the relationship between serum cholesterol and coronary artery disease is not a threshold one, but rather is a continuously gradedone that powerfully affects risk for the great majority of middle-aged American men. ${ }^{10}$ Hypothyroidism is known to be a state of raised lipid profile parameters, and treatment with L-thyroxine therapy has been found to decrease the same. Studies including that done by Alka M. Kanaya et al in 2002, showed elevated thyrotropin level $(>5.5 \mathrm{mIU} / \mathrm{mL})$ was associated with a $9 \mathrm{mg} / \mathrm{dL}(0.23 \mathrm{mmol} / \mathrm{L})$ higher cholesterol level.11 Several prospective studies have demonstrated that after initiation of levothyroxine in those with hypothyroidism, a positive effect is seen on the subsequent lipid profile.12,13 In the present study, the male subset experienced a statistically significant improvement of serum cholesterol and serum triglycerides, whereas the female subset was found to have statistically significant improvement in serum LDL and triglyceride levels.

The cardiovascular system is one of the most important targets of thyroid hormones and is very sensitive to a minimal decrease of circulating thyroid hormones. It has long been recognized that hypothyroidism may cause cardiac pathologies, such as impaired cardiac contractility, decreased cardiac output, increased systemic vascular resistance, and cardiac electrical abnormalities Electrocardiographic changes such as bradycardia low voltage, and varying degrees of heart block are commonly recognized in hypothyroid patients. ${ }^{14,15}$ The cardiac effects of hypothyroidism depend on the severity and duration of the disease and can range from subtle abnormalities to overt and easily recognizable manifestations.

In the present study amongst males, and females alike, bradycardia was the commonest finding during the $1^{\text {st }}$ visit; accounting for totally $48 \%$ of patients in the study population. It has been found that sinus bradycardia of hypothyroidism is suggestive of decreased sympathetic tone, and are seen in 47 $\%$ of all patients at presentation of this study. Decreased $\mathrm{P}$ wave as documented in Braunwald's textbook cardiovascular diseases, was seen in one patient in this study.

Features suggestive of pericardial effusion (Low voltage complexes) were also seen during detection of hypothyroid state in one male and one female patient, but the subsequent ECGs when on treatment were normal in the male, findings in the female persisted. RBBB was seen in one male patient, an ECG finding that persisted throughout follow up period. QT prolongation was documented in one male patient in the present study. But sinus tachycardia and ventricular tachycardia seen in Nikoo's study, but are not found in the present study. ${ }^{15,16}$

\section{Echocardiography Findings}

Initial assessment revealed a normal Echocardiography in $16.7 \%$ males and $33.3 \%$ females. With treatment, totally 43 $\%$ males had a normal ECHO at the end of 6 months treatment, and total of $66.7 \%$ female patients had a normal echo by the end of 6 months. On initial assessment, "raised interventricular septal dimensions" was the commonest finding in males and females alike. In a study by R.Verma in 1995 it was seen that $27 \%$ of patients had diastolic dysfunction. In the present study, diastolic dysfunction initially seen in $21.4 \%$ male patients, decreased to $14.3 \%$ with treatment. In females, $11.1 \%$ had diastolic dysfunction, i. e. 2 out of 18 female patients. This reduced to $5.56 \%$ at the end of 6-month treatment of hypothyroidism. In Dr. R. Verma's study, increased numbers of pericardial effusion and diastolic dysfunction, as well as increased interventricular septal dimensions in both subclinical and overt hypothyroidism were noted. ${ }^{17}$ The overall number of normal Echo reports increased with time and treatment as evidenced by a p value of 0.034 amongst the female patients. Other parameters did not show a significant change with treatment. In several studies, a sensitive measure of myocardial contractility, the ratio of preejection period to left ventricular ejection time (PEP: LVET ) was shown to improve significantly in patients with subclinical hypothyroidism who were treated with levothyroxine, compared with patients who were treated with placebo. ${ }^{18}$ 


\section{CONCLUSIONS}

32 newly diagnosed hypothyroid patients presented with an abundance of features suggestive of raised cardiovascular morbidity in the form of raised fasting lipids, bradycardia, raised interventricular septal dimensions and diastolic dysfunction. Treatment in the form of levothyroxine therapy improved all the above-mentioned parameters to a small extent, and other studies have in fact, shown a long-term benefit. This study proves the need to diagnose and treat hypothyroidism at an early stage. It should be done on a largescale with follow up for a prolonged duration to reiterate the benefits of treatment of hypothyroidism.

\section{REFERENCES}

[1] Cáp J. Hypothyroidism substitution and adrenal insufficiency in diabetic patients. Vnitr Lek 2009;55(4):371-4.

[2] Constant EL, Adam S, Seron X, et al. Hypothyroidism and major depression: a common executive dysfunction? J Clin Exp Neuropsychol 2006;28(5):790807.

[3] Ord WM. Report of a committee of the Clinical Society of London nominated December 14, 1883, to investigate the subject of myxoedema. Trans Clin Soc Lond 1888;21:1-215.

[4] Gillett M. Subclinical hypothyroidism: subclinical thyroid disease: scientific review and guidelines for diagnosis and management. JAMA 2004;291:228-38.

[5] Woeber KA. Update on the management of hyperthyroidism and hypothyroidism. Arch Intern Med 2000;160(8):1967-71.

[6] Lambert EH, Underdahl LO, Beckett S, et al. A study of the ankle jerk in myxedema. J Clin Endocr Metab 1951;11(10):1186-205.

[7] Canaris GJ, Steiner JF, Ridgway EC. Do traditional symptoms of hypothyroidism correlate with biochemical disease? J Gen Intern Med 1997;12(9):54450 .

[8] Golding DN. Hypothyroidism presenting with musculoskeletal symptoms. Ann Rheum Dis 1970;29(1):10-4.
[9] Imtiaz F, Fatima T, Ayoob Z. Importance of thyroid. Journal of the Dow University of Health Sciences 2009;3(2):82-5.

[10] Stamler J, Neaton JD. The Multiple Risk Factor Intervention Trial (MRFIT) - importance then and now. JAMA 2008;300(11):1343-5.

[11] Kanaya AM, Harris F, Volpato S, et al. Association between thyroid dysfunction and total cholesterol level in an older biracial population: the health, aging and body composition study. Arch Intern Med 2002;162(7):773-9.

[12] Monzani F, Carraccio N, Kozakowa M, et al. Effect of levothyroxine replacement on lipid profile and intimamedia thickness in subclinical hypothyroidism: a double-blind placebo-controlled study. J Clin Endocrinol Metab 2004;89(5):2099-106.

[13] Perez A, Cubero J, Sucunza N, et al. Emerging cardiovascular risk factors in subclinical hypothyroidism: lack of change after restoration of euthyroidism. Metabolism 2004;53(11):1512-5.

[14] Sanders V. Neurologic manifestations of myxedema. N Engl J Med 1962;266:599-603 concl.

[15] Santos AD, Miller RP, Mathew PK, et al. Echocardiographic characteristization of the reversible cardiomyopathy of hypothyroidism. Am J Med 1980;68(5):675-82.

[16] Taddei S, Caraccio N, Virdis A, et al. Impaired endothelium-dependent vasodilatation in subclinical hypothyroidism: beneficial effect of levothyroxine therapy. J Clin Endocrinol Metab 2003;88(8):3731-7.

[17] Amidi M, Leon DF, DeGroot WJ, et al. Effect of the thyroid state on myocardial contractility and ventricular ejection rate in man. Circulation 1968;38(2):229-39.

[18] Longo D, Fauci A, Kasper D, et al. Disorders of thyroid gland. Chapter - 341. Harrison's Principles of internal medicine. $18^{\text {th }}$ edn. The McGraw-Hill Companies, 2011. 